\title{
Cardiac effects of panhypopituitarism in a 71-year-old woman
}

\author{
Bradley Hayley MD, David Birnie MB ChB MD, Benjamin J.W. Chow MD
}

Competing interests: Benjamin Chow has received research grant support through his institution from GE Healthcare and education support from TeraRecon. No other competing interests were declared.

This article has been peer reviewed.

\section{Correspondence to:} Benjamin Chow,

bchow@ottawaheart.ca

CMAJ 2014. DOI:10.1503 /cmaj.111610
1 71-year-old woman presented to the emergency department with confusion, general fatigue and a history of falls over the preceding several weeks. She had no recent symptoms of infection nor any discomfort in her chest. Her medical history included type 2 diabetes mellitus, hypertension and deep vein thrombosis.

On physical examination, the patient looked unwell. She had multiple ecchymoses and sparse hair covering her armpits and eye brows. Her heart rate was 70 beats/min, and her blood pressure was 90/50 mm Hg. She was afebrile. Her cardiac examination was unremarkable, with no extra heart sounds or substantial murmurs. A 12-lead electrocardiogram (ECG) showed a prolonged QT interval with biphasic ST segments in the inferolateral leads, which was suspicious for an underlying metabolic process (Figure 1A). Laboratory investigations showed elevated levels of cardiac troponin I $(11.9$ [normal < 0.04] $\mu \mathrm{g} / \mathrm{L})$ and creatinine kinase (3602 [normal 20-160] IU/L).

After several hours, the patient's condition deteriorated acutely. Her hypotension worsened, requiring inotropic support with norepinephrine. Despite the drop in her blood pressure, the patient did not have a tachycardic response. A subsequent ECG showed more pronounced STsegment elevation (Figure 1B). A coronary angiogram showed normal coronary arteries with severe left ventricular dysfunction (Figure 2). After the angiogram, the patient had recurrent sustained monomorphic ventricular tachycardia at a rate of 156 beats/min (Figure 1C), which was resistant to antiarrhythmics. Electrical cardioversion achieved transient sinus rhythm, with reinitiation of ventricular tachycardia via premature ventricular contractions. Amiodarone and vasopressin were administered intravenously, all inotropic agents associated with arrhythmogenicity were stopped, and there were no further episodes of ventricular tachycardia.

An echocardiogram with contrast agent confirmed severe left ventricular dysfunction with an ejection fraction of $25 \%$ (normal $\geq 55 \%$ ) and no substantial valvular abnormalities. Laboratory investigations for cardiomyopathy and myocarditis showed a thyroid-stimulating hormone level of 0.18 (normal 0.3-5.6) $\mathrm{mU} / \mathrm{L}$, a triiodothyronine level (T3) of 2.8 (normal 3.3-6.0) pmol/L and a random morning cortisol level of 87 (normal 185624) nmol/L, which suggested panhypopituitarism. Treatment with hydrocortisone and thyroxine (T4) was started, which resulted in marked improvement in her hemodynamics and cessation of vasopressors within 24 hours. Magnetic resonance imaging of the patient's brain showed a large cyst of the pituitary stalk causing mass effect, with flattening and destruction of the pituitary gland.

After three weeks of hormone replacement, the patient had complete recovery of her left ventricular function, with no regional wall motion abnormalities. After consulting an endocrinologist, we thought that the patient would not benefit from a surgical resection and that her condition should be treated medically. The patient was subsequently discharged from hospital; her discharge medications included levothyroxine and hydrocortisone.

\section{Discussion}

Ischemic heart disease is the most common cause of ventricular tachycardia and left ventricular dysfunction, but the differential diagnosis is extensive. Systolic impairment may result from incessant tachycardia, viral infections or myocarditis, long-standing hypertension, inheritable conditions, alcohol toxicity and exposure to chemotherapeutic agents. ${ }^{1}$ In addition, cardiomyopathy from 
endocrine causes should be considered in patients with unexplained ventricular dysfunction.

\section{Endocrine causes of left ventricular dysfunction}

Diabetes mellitus is the most common endocrine disorder influencing the development of heart failure, with a population attributable risk of $6 \%$ for men and $12 \%$ for women. ${ }^{2}$ Both coronary artery disease and diastolic dysfunction contribute to the development of heart failure caused by diabetes. Unlike in many other endocrine disorders, treatment of the underlying deficiency will not resolve the damage if myocardial performance is impaired as a result of diabetes mellitus.

Sparse literature exists to identify patients at risk for left ventricular dysfunction caused by other endocrine disorders. The burden of disease required to cause myocardial impairment has not been quantified in most endocrinopathies; their impact on the prevalence of heart failure is unclear but appears to be low. Despite their low incidence, many endocrine disorders can cause abnormal lipid and glucose metabolism that can contribute to atherosclerosis, myocardial ischemia and left ventricular dysfunction. For example, subclinical hypothyroidism, hyperthyroidism and Cushing syndrome have all been implicated in the genesis of coronary artery disease. Their contribution to plaque formation

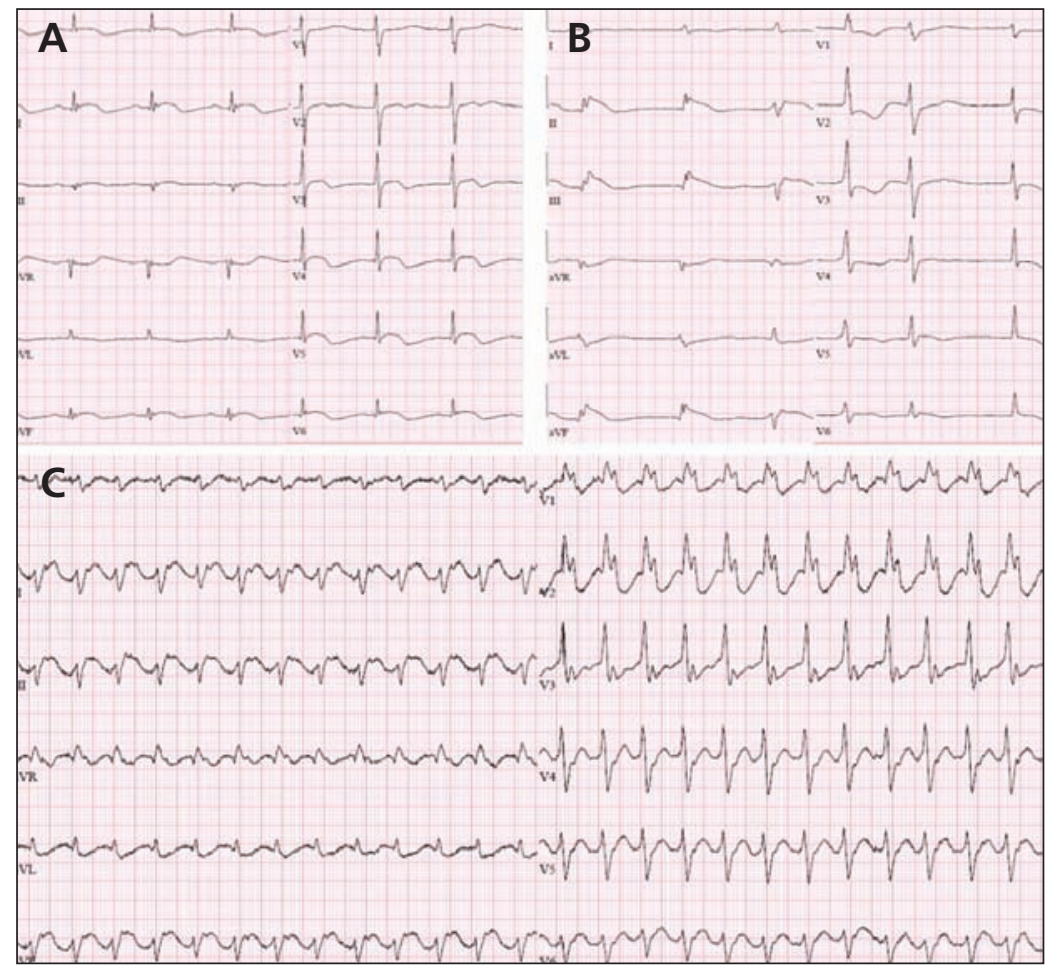

Figure 1: Electrocardiogram (ECG) results from a 71-year-old woman who presented with a recent history of confusion, fatigue and falls. (A) The ECG at presentation showed signs of a metabolic process, with a prolonged QT interval and diffuse ST-segment abnormality. (B) A subsequent ECG showed results more consistent with an acute ischemic insult, with ST-segment elevation in the inferior leads and reciprocal changes in leads I, aVL, V1 and V2. (C) An ECG after angiography showing monomorphic ventricular tachycardia at a rate of 156 beats/min.

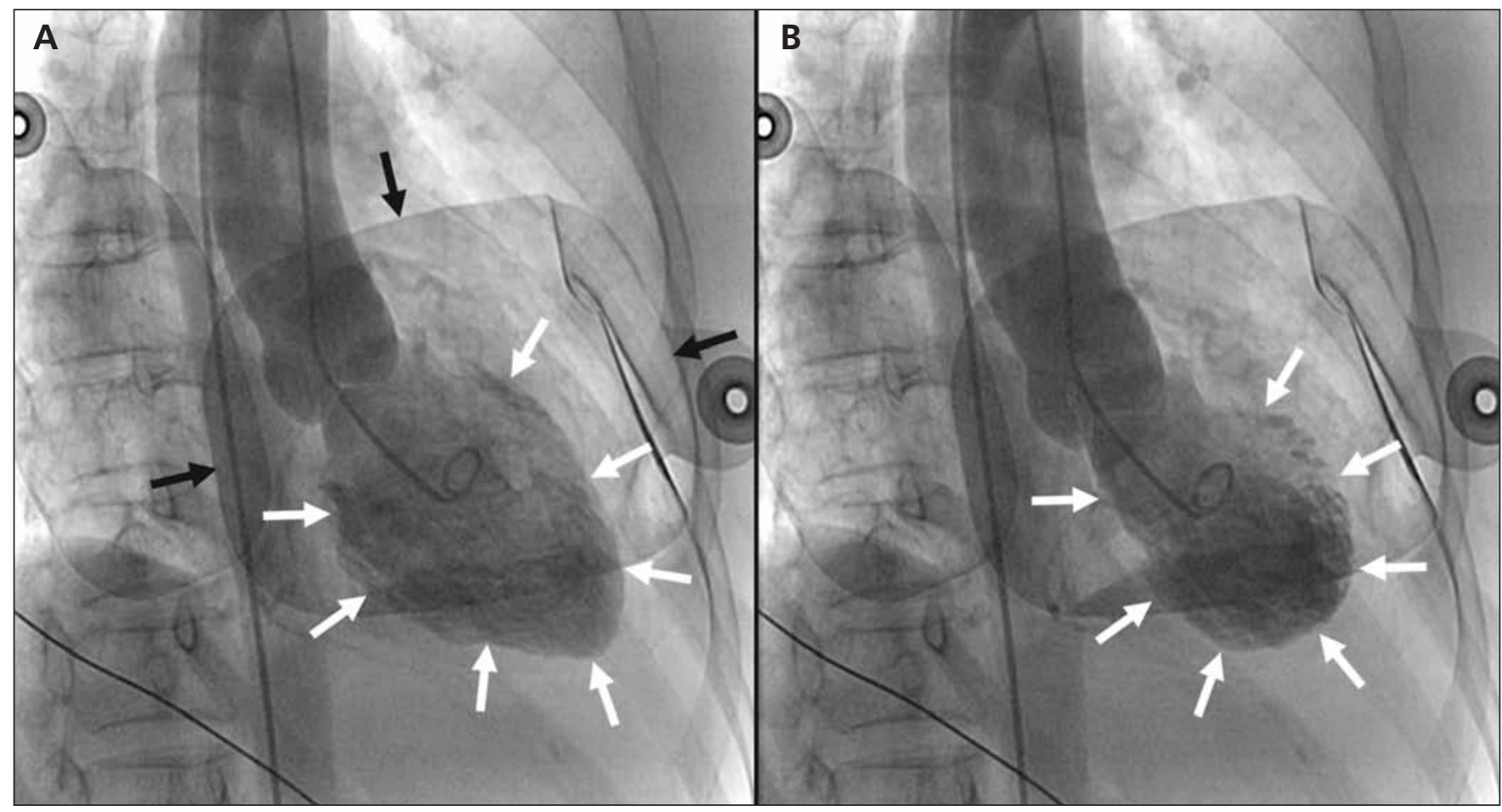

Figure 2: Cardiac ventriculography in the same patient, in the right anterior oblique projection. Comparison of the end-diastolic volume ( $A$, white arrows) with the end-systolic volume (B, white arrows) shows a marked reduction in ejection fraction. Defibrillator pads seen overlying the chest (black arrows) were applied because of runs of nonsustained ventricular tachycardia. 
appears to be attenuated through appropriate recognition and treatment. ${ }^{3.4}$

Thyroid disease is common in the community, particularly among older women. The prevalence of subclinical hypothyroidism is estimated to be as high as $10 \%$ in this group, placing them at potentially higher risk for cardiac complications without the development of overt symptoms. ${ }^{4}$ Both hyperthyroidism and hypothyroidism can cause clinically significant left ventricular dysfunction that occurs through different mechanisms independent of their atherosclerotic contribution. Excess T4 production may result in sinus tachycardia or atrial fibrillation that can lead to tachycardia-induced cardiomyopathy. Con-

\section{Box 1: Electrocardiographic characteristics of various endocrine and} metabolic conditions ${ }^{3,9,10}$

Hyperthyroidism

- Atrial fibrillation, flutter or sinus tachycardia

- High amplitude or notched P waves

- Signs of left ventricular hypertrophy

- Intraventricular conduction delays and atrioventricular block Hypothyroidism

- Bradycardia

- Prolonged QT interval

- Torsades de pointes

- Low-voltage QRS complexes

- Abnormal ST segment (e.g., depression, inversion, elevation)

- Intraventricular conduction delays and atrioventricular block

Adrenal insufficiency

- Bradycardia

- Prolonged QT interval

- Low-voltage QRS complexes

- Flat or inverted T waves

Hypercalcemia

- Shortened QT interval

- Wide T wave

- Prolonged PR interval

Hyperkalemia

- Small P wave

- Peaked T waves

- Wide QRS complex and atrioventricular block

- Sinusoidal pattern

Pheochromocytoma

- Sinus tachycardia

- Ectopic atrial and ventricular beats

- Left ventricular hypertrophy

- Prolonged QT interval

- Abnormal ST segment (e.g., depression, inversion, elevation)

Cushing syndrome

- Left ventricular hypertrophy

- Right ventricular hypertrophy

- Increased heart rate-corrected QT dispersion versely, T4 deficiency results in bradycardia, decreased stroke volume and abnormal myocardial glucose utilization, which can contribute to abnormal cardiac function. Intramyocardial mucopolysaccharide deposition, myocardial edema and pericardial effusion associated with hypothyroidism may also prove detrimental to cardiac function. ${ }^{4}$ However, the syndromes of myocardial dysfunction related to hyperthyroidism and hypothyroidism are almost universally reversible when recognized and treated appropriately. ${ }^{4}$ Therefore, assessing thyroid function (by measuring thyroid-stimulating hormone, $\mathrm{T} 4$ and T3 levels) in a patient with undifferentiated left ventricular dysfunction is essential and recommended by the Canadian Cardiovascular Society's consensus statement on the diagnosis and management of heart failure. ${ }^{5}$

Adrenal crisis (i.e., with a profound glucocorticoid deficiency) is a well-documented cause of circulatory collapse that often results in a high cardiac output state. Although its association with substantial impairment in left ventricular systolic function and cardiogenic shock is rare and not well understood, hormone replacement with glucocorticoids has resulted in rapid resolution of the resulting hypotension and left ventricular dysfunction. ${ }^{6}$ Glucocorticoid overproduction, as in Cushing syndrome, may also result in adverse cardiovascular outcomes through dysfunctional glucose metabolism, hypertension and dyslipidemia. Cardiovascular disease is a leading cause of death and morbidity in overt Cushing syndrome, but its risk may be attenuated through recognition and treatment. ${ }^{3}$ Excess adrenal function, such as in pheochromocytoma, has also been linked to left ventricular dysfunction through excess production of catecholamines. A recent review has suggested that left ventricular dysfunction and Takotsubo cardiomyopathy (apical ballooning syndrome) can occur in about $11 \%$ of catecholamine-producing adrenal tumours, with all cases returning to normal within three days after medical or surgical correction. ${ }^{7}$ Routine screening for adrenal diseases is not recommended by the Canadian Cardiovascular Society guidelines, ${ }^{5}$ but it should be performed if there is clinical evidence to support the diagnosis.

Two cases of cardiomyopathy induced by panhypopituitarism have been reported previously as associated with Sheehan syndrome (i.e., postpartum hypopituitarism). Although both patients had severe left ventricular dysfunction and shock, treatment with glucocorticoids and thyroxine resulted in rapid hemodynamic stabilization and complete reversal of left ventricular dysfunction within seven to nine months. ${ }^{8}$ Similar results were seen in our patient, with hydrocortisone and 
T4 replacement resulting in withdrawal of inotropic therapy within 24 hours, although her left ventricular function returned within a much shorter timeframe (i.e., three weeks). Given the cardiac depressor effects of both hypothyroidism and adrenal insufficiency, it is not surprising that this syndrome can result in cardiovascular collapse. Unlike in previously described cases, our patient's panhypopituitarism was secondary to pituitary compression rather than postpartum hemorrhage and infarction. Although magnetic resonance imaging was helpful in determining the cause of our patient's condition, the basis of diagnosing panhypopituitarism is mainly biochemical and can be challenging.

\section{ECG characteristics of endocrine disorders}

Metabolic and hormonal derangements often manifest in abnormal ECG patterns that can affect any segment of the recording (Box 1) ${ }^{9,10}$ Hypothyroidism is often associated with sinus bradycardia, low-voltage QRS complexes, prolonged QT intervals and abnormal ST segments. Our patient's ECG showed the latter two patterns. Low voltage is often a consequence of coexisting pericardial effusion, myocardial edema and intramyocardial mucopolysaccharide deposition. ${ }^{9}$ Abnormalities of the ST segment are common and can manifest as depression, inversion or elevation that may be mistaken for myocardial ischemia or an acute infarction, as in our patient's case. Such patterns can be diagnostically challenging when patients present with signs of left ventricular dysfunction and elevated cardiac enzymes. Although cardiac troponin is highly sensitive, it is not specific for acute coronary syndromes, because it can be elevated for both cardiac and noncardiac processes. ${ }^{11}$ Ventricular arrhythmias, such as torsade de pointes, are also associated with hypothyroidism because of the condition's propensity to cause bradycardia and prolong the QT interval.

In contrast, sinus tachycardia, atrial flutter or atrial fibrillation may suggest elevated thyroid function. High amplitude or notched $\mathrm{P}$ waves may be noted in hyperthyroidism, and the QRS complexes often show signs of left ventricular hypertrophy. Intraventricular conduction delays and atrioventricular block may be associated with both hyperthyroidism and hypothyroidism. ${ }^{9}$

Left and right ventricular hypertrophy may be manifestations of Cushing syndrome, but none is specific. Recent evidence suggests that heart rate-corrected QT (QTc) dispersion may be independently prolonged in Cushing disease and may predict negative outcomes. ${ }^{3}$ However, this measure is not readily calculated from the ECG, and its clinical applicability is yet to be proven. ${ }^{3}$

Changes on ECG associated with adrenal insufficiency are similar to findings seen in hypothyroidism. Flat or inverted $\mathrm{T}$ waves are common, as are low voltages in the limb leads, sinus bradycardia and QT prolongation. Lengthening of the PR interval is frequently associated with hypocortisolism, but advanced atrioventricular block is not commonly seen. Hyperkalemia and hypercalcemia can result from adrenal insufficiency, and their associated ECG findings may be seen (Box 1). ${ }^{9} 10$ Given that panhypopituitarism often manifests with multiple hormonal aberrations, ECG features may resemble many of the previously described endocrinopathies. ${ }^{9}$

\section{Conclusion}

Left ventricular dysfunction has a large differential diagnosis and is not synonymous with coronary artery disease. Endocrine disorders may result in adverse cardiovascular function by contributing to atherosclerosis and altered myocardial metabolism. Although the results of an ECG may be abnormal in various metabolic derangements, the diagnosis may remain unclear. Additional history and physical examination should be sought to support an endocrine cause in patients with idiopathic cardiac dysfunction. Investigations may include screening for thyroid dysfunction because of its high prevalence and treatability, although less common conditions may be investigated if clinical suspicion is high.

\section{References}

1. McMurray JJ. Systolic heart failure. N Engl J Med 2010;362:228-38.

2. Kannel WB. Incidence and epidemiology of heart failure. Heart Fail Rev 2000;5:167-73.

3. Alexandraki KI, Kaltsas G, Vouliotis A, et al. Specific electrocardiographic features associated with Cushing's disease. Clin Endocrinol (Oxf) 2011;74:558-64.

4. Klein I, Ojamaa K. Thyroid hormone and the cardiovascular system. N Engl J Med 2001;344:501-9.

5. Arnold JM, Liu P, Demers C, et al. Canadian Cardiovascular Society consensus conference recommendations on heart failure 2006: diagnosis and management. Can J Cardiol 2006;22:23-45.

6. Mekontso-Dessap A, Marrache D, Vieillard-Baron A. Acute adrenal insufficiency complicated by cardiogenic shock. Heart 2005;91:e31.

7. Park JH, Kim KS, Sul JY, et al. Prevalance and patterns of left ventricular dysfunction in patients with pheochromocytoma. J Cardiovasc Ultrasound 2011;19:76-82.

8. Bao SS, Fisher SJ. Repairing a "broken heart" with hormone replacement therapy: case report of cardiogenic shock due to undiagnosed pituitary insufficiency. Endocr Pract 2012;18:e26-31.

9. Surawicz B, Mangiardi ML. Electrocardiogram in endocrine and metabolic disorders. Cardiovasc Clin 1977;8:243-66.

10. Diercks DB, Shumaik GM, Harrigan RA, et al. Electrocardiographic manifestations: electrolyte abnormalities. J Emerg Med 2004;27:153-60.

11. Inbar R, Shoenfeld Y. Elevated cardiac troponins: the ultimate marker for myocardial necrosis, but not without a differential diagnosis. Isr Med Assoc J 2009;11:50-3.

Affiliations: Division of Cardiology (Hayley), Memorial University, St. John's, NL; Division of Cardiology (Birnie, Chow), University of Ottawa Heart Institute, Ottawa, Ont.

Contributors: All of the authors contributed to the writing and revising of the manuscript and approved the final version submitted for publication. 